\title{
Tracer transport along and across coherent jets in two-dimensional turbulent flow
}

\author{
By K. SHAFER SMITH \\ Center for Atmosphere Ocean Science, Courant Institute of Mathematical Sciences, \\ New York University, 251 Mercer St., New York, NY 10012, USA
}

(Received 19 August 2005 and in revised form 21 September 2005)

\begin{abstract}
A theory for the transport of a tracer in flow dominated by turbulence and jets is developed and tested. Such a system can be taken as a model either for the stirring of true tracers in the atmosphere and ocean, or, less obviously, for the stirring of baroclinic potential vorticity by non-zonal flow in the ocean. The flow is generated by two-dimensional turbulence with a mean vorticity gradient $(\beta)$ and forced randomly and isotropically at small scales. The tracer transported by the flow is forced by a mean tracer gradient that is arbitrarily oriented with respect to the mean vorticity gradient. Such a tracer can be decomposed into two independent tracers: one forced by a gradient that is parallel to the vorticity gradient (and so is stirred across jets), and another that is forced by a mean gradient that is perpendicular to the mean vorticity gradient (and so is stirred along jets). The turbulent transport of the tracer across jets has been discussed in previous research, and is well-described by mixing-length theory. Here it is shown that the transport of the tracer along the jets is described by shear dispersion, but with a diffusivity determined by the across-jet mixing. Even at only moderate levels of anisotropy, the along-jet transport is much larger than the corresponding across-jet transport, consistent, for example, with observations from surface floats in the Pacific.
\end{abstract}

\section{Introduction}

The statistical description of tracer transport in turbulent flows is a prevalent concern in atmospheric and oceanic fluid dynamics, as well as other branches of environmental science. In the parameterization of turbulent tracer transport in coarseresolution numerical climate models, for example, the problem is often treated as isotropic down-gradient diffusion, regardless of the complexity of the advecting flow. This is strictly appropriate only when the advecting flow is isotropic with Gaussian randomness. Atmospheric and oceanic flows, by contrast, are typically anisotropic, intermittent, and inhomogeneous. Attempts to include the effects of mean flow anisotropy in a parameterization, as in Smith \& Gent (2004), are very recent and largely kinematic in nature.

The present paper seeks to develop the underlying principles of an anisotropic tracer flux parameterization from a fundamental perspective. In particluar, we discuss a theory for the diffusive transport in a flow dominated by jets in a turbulent background, typical, for example, of $\beta$-plane turbulence. This problem was investigated by Holloway \& Kristmannsson (1984) and Bartello \& Holloway (1991), but no theory was provided in these papers for the effective diffusivity in the along-jet direction. The transport in the cross-jet direction was analysed by these authors with some success, but their analyses relied on cumbersome statistical turbulence closure models, such as 
the Test Field Model used by Bartello \& Holloway (1991). Holloway (1986) proposed a simplification of the scale found in a similar model (the Markovian Random Coupling Model), but even the simplification requires two non-dimensional fit parameters, as well other information in order to work as a usable closure. More recently Smith et al. (2002) demonstrated that, even in the presence of jets, if the correct velocity and length scales are used, the cross-jet transport is well-described by a mixing-length theory, providing a far simpler method of estimation and prediction. A similar scaling is derived by Held \& Larichev (1996) and shown to be relevant to atmospheric heat transport by Barry, Craig \& Thurburn (2002).

Intuitively, the transport in the direction of a jet should exceed that in the direction across the jet. This is found, for example, in observations of satellite-tracked drifting buoys in the tropical Pacific (Bauer, Swenson \& Griffa 2002), where the surface velocity field is dominated by zonal flows. The authors' analysis of those data reveals zonal diffusivities that are about 7 times larger than meridional diffusivities, consistent with intuition.

The primary concern of this paper is the development of a predictive theory for the along-jet transport. It is shown that the along-jet transport is a shear-dispersion process, controlled by the diffusivity of the background turbulence. In the original shear dispersion problem treated by Taylor (1953), it is the molecular diffusivity that acts to disrupt pair displacement flights along the jet axis, but the mechanism is fundamentally the same as in the present case. A previous theory for the scaling of the turbulent jets is combined with the tracer transport theory to derive a closure for the total transport (the diagonal terms of a diffusivity tensor). The theory is shown to describe, with reasonable accuracy, the results of a series of numerical simulations of tracer advection by two-dimensional $\beta$-plane turbulence, over a range of flow anisotropy levels.

It is worth noting some of the applications of such a theory. In the ocean, density is approximately given by a linear combination of temperature and salinity. A second linear combination of temperature and salinity (called spice), for which the change in density associated with each component cancels, can be constructed, and this field effectively acts as a true passive tracer on the range of scales at which mesoscale eddies are dominant (Klein, Treguier \& Hua 1998). If the flow is anisotropic on the same scales, the transport of spice will itself be anisotropic and isopycnal, and might be described by the theory presented here.

A less obvious application concerns the transport of baroclinic potential vorticity in the ocean. In baroclinic turbulence, when the mean flow is not zonal (a situation common in the ocean), the gradient of the mean baroclinic potential vorticity is not parallel to the effective mean vorticity gradient, and so the baroclinic potential vorticity is stirred by velocity components both parallel to and perpendicular to the effective vorticity gradient. Because baroclinic energy generation is given by the integrated flux of potential vorticity (see e.g. Larichev \& Held 1995), one should thus expect to find higher eddy energy in regions of non-zonal flow. Such an increase in eddy energy in the presence of non-zonal flow in numerical models is observed and discussed by Dubus (1999), Spall (2000) and Arbic \& Flierl (2004). The theory presented here can explain these results, but this application will be presented elsewhere.

The basic model and a preliminary numerical simulation are described in $\S 2$. The results of the simulation motivate the construction and solution of an analytic model for the along-jet transport, presented in $\S 3$. The analytic model for the tracer presumes that the flow is given, but in general this is not the case. Scaling theories for geostrophic turbulence are used in $\S 4$ to predict the flow statistics, resulting in a 
closure theory for the diffusivity. The theory is tested against simulations in $\S 5$, and the paper concludes with $\S 6$.

\section{Basic model and a preliminary simulation}

We consider a flow governed by the two-dimensional vorticity equation,

$$
\zeta_{t}+J(\psi, \zeta+\beta y)=\mathscr{F}-r \zeta+\mathscr{D} \zeta
$$

where $\zeta=\hat{z} \cdot \nabla \times \boldsymbol{u}=\nabla^{2} \psi$ is the vorticity, $\psi$ is the streamfunction, $(u, v)=\left(-\psi_{y}, \psi_{x}\right)$ is the velocity, $\beta$ is the mean vorticity gradient, $\mathscr{F}$ is a small-scale, isotropic random forcing, $\mathscr{D}$ is a linear (enstrophy) dissipation operator and $r$ is a linear drag coefficient.

The flow advects a tracer with a steady mean gradient

$$
c_{t}+\boldsymbol{u} \cdot \nabla(c+\boldsymbol{\Gamma} \cdot \boldsymbol{x})=\mathscr{D} c
$$

where $c$ is the deviation tracer concentration from the constant mean gradient $\boldsymbol{\Gamma}=(\Gamma, \Lambda)$, and $\mathscr{D}$ is a scale-selective linear dissipation operator.

Given two independent tracers $\chi$ and $\phi$ that respectively satisfy

$$
\begin{gathered}
\chi_{t}+\boldsymbol{u} \cdot \nabla \chi+u=\mathscr{D} \chi, \\
\phi_{t}+\boldsymbol{u} \cdot \nabla \phi+v=\mathscr{D} \phi,
\end{gathered}
$$

the original tracer $c$ can be reconstructed from

$$
c=\Gamma \cdot \chi
$$

where $\chi=(\chi, \phi)$ (see e.g. Reed \& German 1965; Majda \& Kramer 1999).

Given the direction of the vorticity gradient in (2.1), one should expect a qualitative difference in the dynamics of $(2.3 a)$ and $(2.3 b)$. In the presence of $\beta$, it is known that the steady-state velocity field will be characterized by a series of stable alternating jets oriented perpendicular to the gradient, along the $x$-direction, embedded in a background of small-scale isotropic turbulence (e.g. Rhines 1975; Maltrud \& Vallis 1991). In other words, $u$ will be dominated by coherent and elongated structures in the $x$-direction, while $v$ will be isotropic and random.

Making the assumption of double periodicity, the variance equations for the two orthogonal tracers are

$$
\begin{aligned}
& \partial_{t}\left\langle\chi^{2}\right\rangle / 2=D_{\chi}+\langle\chi \mathscr{D} \chi\rangle, \\
& \partial_{t}\left\langle\phi^{2}\right\rangle / 2=D_{\phi}+\langle\phi \mathscr{D} \phi\rangle,
\end{aligned}
$$

where $D_{\chi}=-\langle u \chi\rangle, D_{\phi}=-\langle v \phi\rangle$, and \langle\rangle denotes a space (or homogeneous) average. Thus the variance of $\chi$ is forced by correlations with the coherent zonal velocity field, while the variance of $\phi$ is forced by correlations with the random isotropic meridional flow. We will refer to $D_{\chi}$ as the 'along-jet' diffusivity and $D_{\phi}$ as the 'across-jet' diffusivity. $\dagger$ Mixing-length theory can be used with some skill to predict $D_{\phi}$ (Smith et al. 2002), while no prior theory for $D_{\chi}$ is known to the author.

A preliminary numerical simulation was performed using a standard pseudospectral model to solve (2.1) and (2.3). The model details are summarized in Smith et al. (2002). The preliminary simulation has $256^{2}$ equivalent grid points, or a maximum isotropic wavenumber $k_{\max }=127$. The forcing is localized to wavenumbers $k_{f}=$ 80 and the small-scale dissipation is effected by an exponential cutoff filter, and

$\dagger$ In a flux gradient relationship for $\chi$, for example, the transport $\langle u \chi\rangle=-D \mathrm{~d} \bar{\chi} / \mathrm{d} x$, but here the mean gradient $\mathrm{d} \bar{\chi} / \mathrm{d} x=1$, so the diffusivity $D=-\langle u \chi\rangle$. 
(a)

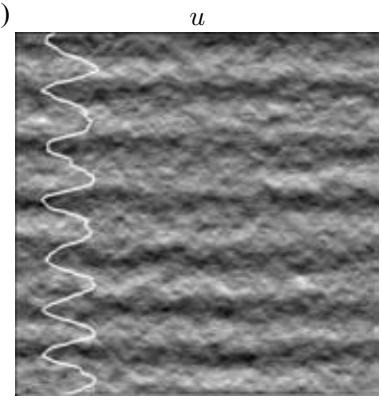

(d)

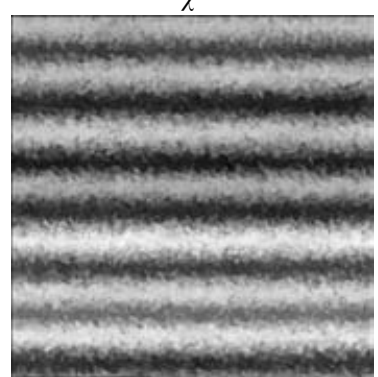

(b)

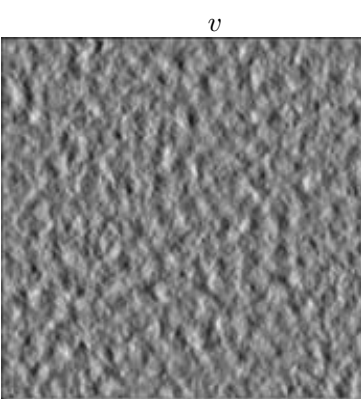

(e)

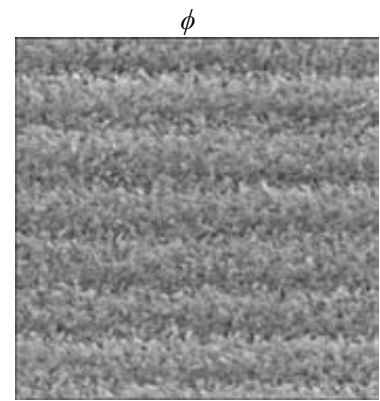

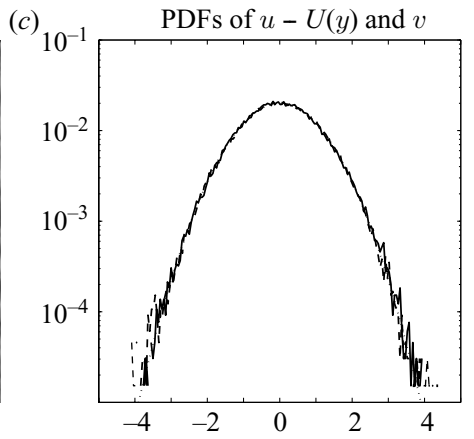

$(f)$

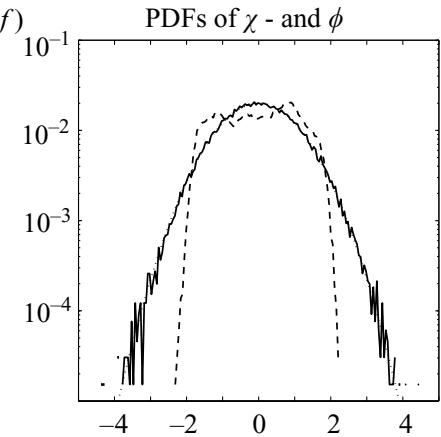

FIGURE 1. Snapshots of $(a) u,(b) v,(d) \chi$ and $(e) \phi$ from the preliminary simulation with $\beta=1000$ and $r=0.2$. The overlayed white curve in $(a)$ is the zonal average $U=\bar{u}$. (c) The PDFs of $v$ (solid line) and $u-U(y)$ (dashed line), and $(f)$ the PDFs of $\phi$ (solid line) and $\chi$ (dashed line). Also plotted are Gaussian probability distributions (dotted line) with same variances and means as $v$ and $\phi$, respectively.

restricted to wavenumbers $k>85$. The forcing is normalized to yield unit overall energy generation after dissipation by the filter $\mathscr{D}$. Thus where ever it appears from here on, the upscale energy transfer rate $\epsilon$ is taken to be 1 . Unless stated otherwise, all parameter values are given in 'spectral units', with $L=2 \pi$ (in these units, $k=1$ is the wavenumber of a wave that just fills the domain). The preliminary simulation used $\beta=1000$ and $r=0.2$.

Snapshots of the two components of the velocity field, and the two tracer fields $\chi$ and $\phi$ are shown in figure 1. These reveal the expected presence of zonal jets in $u$ (its zonal mean is overlayed in white on panel $a$ ) and corresponding coherence in $\chi$, as well as isotropic randomness in $v$. The $\phi$ field faintly shows the presence of jets, since it is advected by both $u$ and $v$. Nevertheless, its variance is directly forced only by $v$. The number of jets in the flow, or jet wavenumber, will be discussed in $\S 4$.

Panels $(c)$ and $(f)$ of figure 1 show the probability density functions (PDFs) of the velocity and tracer fields, respectively. Notice particularly that the PDF for $\phi$ is nearly Gaussian, consistent with the success of mixing-length theory in describing the across-jet diffusivity (in other words, the cross-jet tracer concentration is consistent with the solution of a diffusion equation with constant turbulent diffusivity). The PDFs for $v$ and $u-U(y)$ (where $U(y)$ is the average of $u$ in $x$ and $t$ ) similarly are nearly Gaussian, and importantly, the PDFs have the same widths, implying the existence of a well-defined background isotropic diffusivity. In the along-jet direction, pairs of particles are driven long distances apart by large-scale shear in the flow. In the absence of any small-scale mixing or diffusion, the dispersion should grow linearly in time without truncation. Thus we expect that the overall tracer variance 
and diffusivity will be much larger for $\chi$ than for $\phi$. In this preliminary simulation, we find $D_{\chi} / D_{\phi}=500$.

\section{A model for the along-jet transport}

The structure of the flow in the preliminary simulation suggests the following simple model: a large-scale mean zonal velocity, independent of $x$ and $t$, embedded in a small-scale isotropic turbulent background whose statistics are described by an inverse cascade process. Denoting the average in $x$ of some function $f$ with an overline, $\bar{f}$, we define

$$
U=\bar{u}, \quad u^{\prime}=u-U .
$$

In the homogeneous system discussed here $\langle u\rangle=0$, and since the background turbulence is assumed isotropic, we define a turbulent velocity scale

$$
v_{\text {turb }}=\left(\left\langle u^{\prime 2}\right\rangle+\left\langle v^{2}\right\rangle\right)^{1 / 2} \text {. }
$$

Since the statistics of the background flow in the preliminary simulation do appear to be stationary, Gaussian and isotropic, we further assume that the turbulence is well-described by a positive turbulent diffusivity

$$
\kappa=D_{\phi} .
$$

Given these assumptions and definitions, consider the advection-diffusion equation in the presence of a large-scale, periodic, smooth mean flow $\boldsymbol{u}=(U(y), 0)$ and a turbulent diffusivity $\kappa$, so that

$$
\chi_{t}-\kappa \chi_{y y}=-U(y)
$$

The lack of coherent structure in the $x$-direction, and the periodicity in that direction allow us to neglect derivatives in $x$. This is a form of the shear dispersion problem of Taylor (1953), also discussed by Young, Rhines \& Garrett (1982). Several related models of this type are considered by Bourlioux \& Majda (2002).

Allowing an arbitrary mean flow structure, we expand it in a Fourier series

$$
U(y)=\sum_{n=-\infty}^{\infty} \hat{U}_{n} \mathrm{e}^{-i k_{n} y}
$$

where $k_{n}=n \pi / L$. Assuming $\chi(t=0)=0$, the model has the solution

$$
\chi=-\kappa^{-1} \sum_{n=-\infty}^{\infty} \frac{\hat{U}_{n}}{k_{n}^{2}}\left(1+\mathrm{e}^{-k_{n}^{2} \kappa t}\right) \mathrm{e}^{-\mathrm{i} k_{n} y} .
$$

At equilibrium $\left(t \gg L^{2} / \kappa\right)$, the diffusivity takes on the constant value

$$
D_{\chi}=-\left\langle U \chi_{\text {eqm }}\right\rangle=\kappa^{-1} \sum_{n=-\infty}^{\infty} \frac{\left|\hat{U}_{n}\right|^{2}}{k_{n}^{2}}
$$

where $\chi_{\text {eqm }}=\lim _{t \rightarrow \infty} \chi$. As in the classic problem, the along-jet diffusion is inversely proportional to the diffusivity, and the tracer is anti-correlated with the velocity. The unique element of the problem here is the association of the diffusivity with the background turbulent cascade via (3.3).

The root-mean-square (RMS) concentration variance also equilibrates to a constant value at large times, given by

$$
\chi_{\mathrm{rms}}=\sqrt{\left\langle\left|\chi_{\mathrm{eqm}}\right|^{2}\right\rangle}=\kappa^{-1} \sqrt{\sum_{n=-\infty}^{\infty} \frac{\left|\hat{U}_{n}\right|^{2}}{k_{n}^{4}}} .
$$


In the limit $\kappa \rightarrow 0$, both $D_{\chi}$ and $\chi_{\text {rms }}$ increase proportional to time $t$ and so never equilibrate.

\section{Scaling theory for turbulent flow on the $\beta$-plane}

Estimates for most of the components of the diffusivity theories discussed above exist in the literature. We shall review and, where necessary, extend them here. The end result will be a scaling theory for the along-jet diffusivity, $D_{\chi}$.

\subsection{Prediction for $L_{j e t}$}

The linear vorticity drag constrains the total energy of the system to be $E=\epsilon / 2 r$ with only minimal distortion of the inertial range of the inverse cascade (Smith et al. 2002). The results reported in the previous reference demonstrate that a good estimate of the inverse jet scale (or peak meridional wavenumber) is given by their equation (6.11), repeated here for convenience:

$$
k_{\mathrm{jet}} \simeq\left(\frac{\mathscr{C}_{\beta} \beta^{2} r}{2 \epsilon}\right)^{1 / 4},
$$

where $\mathscr{C}_{\beta}$ is a non-dimensional parameter (but note that Danilov \& Gryanik 2004 present evidence that this parameter is not universal, and so it should be used with caution). That relation was derived in Smith et al. (2002) in two ways (each method giving a different non-dimensional prefactor), and Danilov \& Gryanik (2004) furthermore suggest the same scaling (but with yet another non-dimensional prefactor) as an upper bound. Using the form of non-dimensional prefactor in (4.1), the estimate $\mathscr{C}_{\beta}=0.2$ seems to fit the simulated data in Danilov \& Gryanik (2004) and Smith et al. (2002), and is close to both the estimate found in Huang, Galperin \& Sukoriansky (2001) and the estimate one can infer from Chekhlov et al. (1996) (both about 0.3). Smith (2004) uses $\mathscr{C}_{\beta}=0.2$ in a local theory that does a reasonable job of describing the jet spacings on Jupiter and Saturn. We will take $\mathscr{C}_{\beta}=0.2$ in this paper.

\subsection{Prediction for $\kappa=D_{\phi}$ and discussion of $v_{t u r b}$ and $\ell_{\text {mix }}$}

Smith et al. (2002) show that the scale on which the jets self-organize (4.1) differs from the scale of the background turbulent mixing (the across-jet transport). The latter is found to be the largest scale at which the flow is isotropic, or smallest scale of anisotropy, given by

$$
k_{\beta}=\left(\frac{\beta^{3}}{\mathscr{C} \epsilon}\right)^{1 / 5}
$$

where $\mathscr{C} \simeq 6$ is the Kolmogorov constant. The RMS isotropic turbulent velocity is then obtained by integrating the $k^{-5 / 3}$ Kolmogorov spectrum up to $k_{\beta}$, giving

$$
v_{\text {turb }} \simeq\left(\frac{3}{2}\right)^{1 / 2}\left(\frac{\mathscr{C}^{3} \epsilon^{2}}{\beta}\right)^{1 / 5}
$$

Equation (4.3) can be multiplied by the inverse of (4.2) to give the diffusivity suggested in equation (7.18) of Smith et al. (2002), but this prediction for the across-jet diffusivity turned out to be about a factor of 2-3 too large. As will be demonstrated in the next section, a better estimate results if one neglects the non-dimensional parameters, 
replacing them with the factor 2 , and is

$$
D_{\phi} \simeq 2\left(\frac{\epsilon^{3}}{\beta^{4}}\right)^{1 / 5} .
$$

An important fact here is that the across-jet diffusivity is predicted to be independent of $r$, as found in Smith et al. (2002, figure 8).

\subsection{Prediction for $U_{r m s}$}

A prediction for the RMS jet velocity in $\beta$-plane turbulence is discussed briefly by Smith et al. (2002) for the limit $U_{0} \gg v_{\text {turb }}$. Here we develop a more complete estimate, accurate in the intermediate range when $U_{0}$ is not much larger than $v_{\text {turb }}$. Again, the energy constraint proves useful, the total energy being

$$
E \simeq \frac{1}{2} U_{0}^{2}+v_{\mathrm{turb}}^{2}=\frac{\epsilon}{2 r}
$$

where $U_{0}=\sqrt{\left\langle U^{2}\right\rangle}$. Expression (4.5) is an approximation due to two implicit assumptions: (i) that $\left\langle u^{\prime} U\right\rangle=0$, and (ii) that $\left\langle u^{\prime 2}\right\rangle=\left\langle v^{2}\right\rangle$. Looking at figure 1(a), we can see that (i) will not generally be true, since correlations between $U$ and $u^{\prime}$ are non-zero. Because of this, assumption (ii) will also be inaccurate. Nevertheless, we proceed, keeping these potential errors in mind. Using relation (4.3) for $v_{\text {turb }}$, this gives us

$$
U_{0}=\left[\frac{\epsilon}{r}-3\left(\frac{\mathscr{C}^{3} \epsilon^{2}}{\beta}\right)^{2 / 5}\right]^{1 / 2} .
$$

In the limit $\beta \rightarrow 0, U_{0}$ apparently becomes imaginary and then diverges, but this is of course not so. The scale $k_{\beta}$ in (4.2) is only valid in the limit that $\beta$ is large enough to yield anisotropic flow. This will be not be the case if the drag is too large or $\beta$ too small, as discussed by Smith et al. (2002), who express this limit in their equation (6.7). A slightly more stringent requirement comes directly from (4.6), which is real and non-zero only if

$$
\beta>\beta_{c}=\left(32 \mathscr{C}^{6}\right)^{1 / 2}\left(\frac{r^{5}}{\epsilon}\right)^{1 / 2} .
$$

The non-dimensional factor in (4.7) is larger by a factor of 10 than the factor in Smith et al. (2002), but because of the weak dependence of $v_{\text {turb }}$ on $\beta$, still allows (4.6) to give a prediction for $U_{0}$ when $\beta$ is so small that almost no anisotropy forms (see $\S 4.4$ for evidence). Note also that: (i) by definition $U=\bar{u} \rightarrow 0$ when no anisotropy is present, and (ii) equivalently, in the same limit of no anisotropy, $v_{\text {turb }} \rightarrow \sqrt{\epsilon / r}$ which sets $U_{0}=0$ via (4.6).

\subsection{Overall prediction for $D_{\chi}$ and discussion of expected dependence}

In combining estimates (4.1), (4.4) and (4.6) with (3.6) to predict the diffusivity, we assume a single mode velocity $U$ to make the correspondence clear. Assuming, for example

$$
U(y)=\hat{U}_{j} \sin \left(k_{j} y+\alpha\right)
$$

where $\alpha$ is the constant phase, we find that

$$
D_{\chi}=\frac{\hat{U}_{j}^{2}}{2 \kappa k_{j}^{2}} .
$$




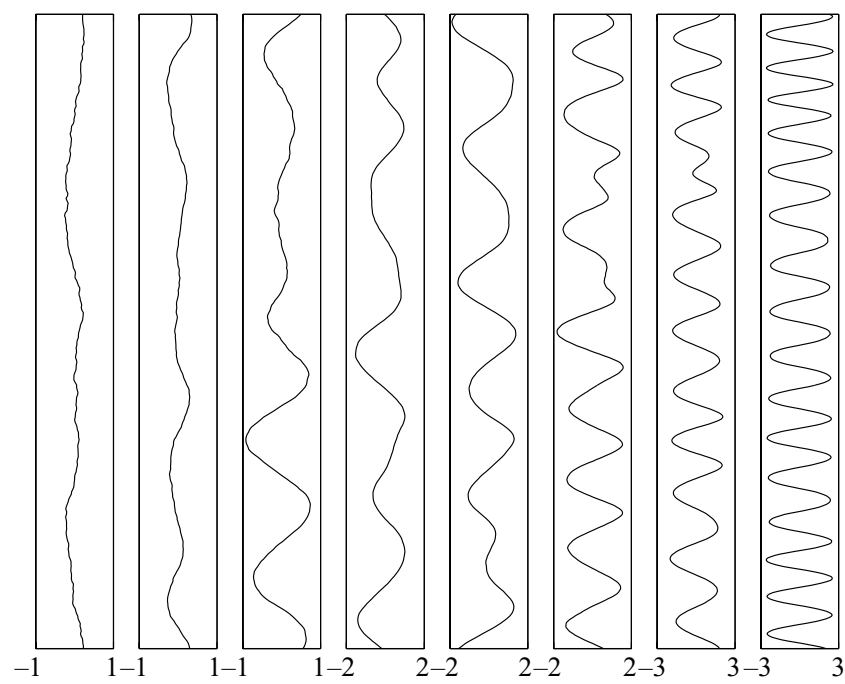

FigURE 2. Zonal- and time-averaged $U(y)=\bar{u}$ for each simulation, with $\beta=50$ on the left and $\beta=4000$ on the right (see beginning of $\S 5$ ). Note that bottom axis is not the same for all plots.

The RMS zonal velocity, predicted by the scaling relation (4.6), is related to the velocity coefficient via $U_{0}=\hat{U}_{j} / \sqrt{2}$. Setting $k_{j}$ equal to $k_{\text {jet }}$ in (4.1) and the diffusivity $\kappa=D_{\phi}$ in (4.4), we then obtain the following prediction for the along-jet diffusivity:

$$
D_{\chi} \simeq A\left[\frac{\epsilon}{r}-B\left(\epsilon^{2} \beta^{-1}\right)^{2 / 5}\right]\left(\epsilon r^{5} \beta^{2}\right)^{-1 / 10},
$$

where $A=\left(\mathscr{C}_{\beta} / 2\right)^{-1 / 2} / 2 \simeq 1.6$ and $B=3 \mathscr{C}^{6 / 5} \simeq 26$. The form of the predicted diffusivity is, for fixed $\epsilon$ and $r$, rapidly increasing at small $\beta$ (near $\beta_{c}$ ), achieves a maximum at moderate $\beta$ (such that $\epsilon / r$ dominates the difference in the brackets), and decreases weakly $\left(\propto \beta^{-1 / 5}\right)$ at large $\beta$.

\section{Numerical investigation of the homogeneous flow statistics}

A series of eight simulations was performed to test the diffusivity scalings presented in $\S 4$. A different value of $\beta(50,100,200,300,500,1000,2000$, and 4000) was used for each simulation. The other parameters were identical to those used in the preliminary simulation, except that the series simulations used a resolution of $512^{2}$, and were forced at wavenumber $k_{f}=160$. Figure 2 shows the zonally averaged zonal velocity $U(y)$ at some point during steady state for each of the simulations. The expected increase in the number and regularity of the jets is apparent.

The across-jet and along-jet effective diffusivities are plotted in figures $3(a)$ and $3(b)$ respectively. Statistics from the simulations are plotted with triangles $(\triangle)$. The solid line in panel $(a)$ is the predicted across-jet diffusivity $\kappa=D_{\phi}(4.4)$. The theory seems to represent the simulated data well. The two curves in panel $(b)$ are as follows. First, as a fundamental test for the shear-dispersion model, equation (3.6) is applied directly, using the spectral transforms of $U(y)$ and the simulated values of $D_{\phi}$ for each run (dotted line with $\times$-marks). This curve appears consistent with the simulated data, providing confirmation of the concept. The solid line in panel $(b)$ represents the overall scaling theory (4.9). All of the theories for all of the constituent parts derived in $\S 4$ must be combined to form the overall scaling theory for the along-jet 

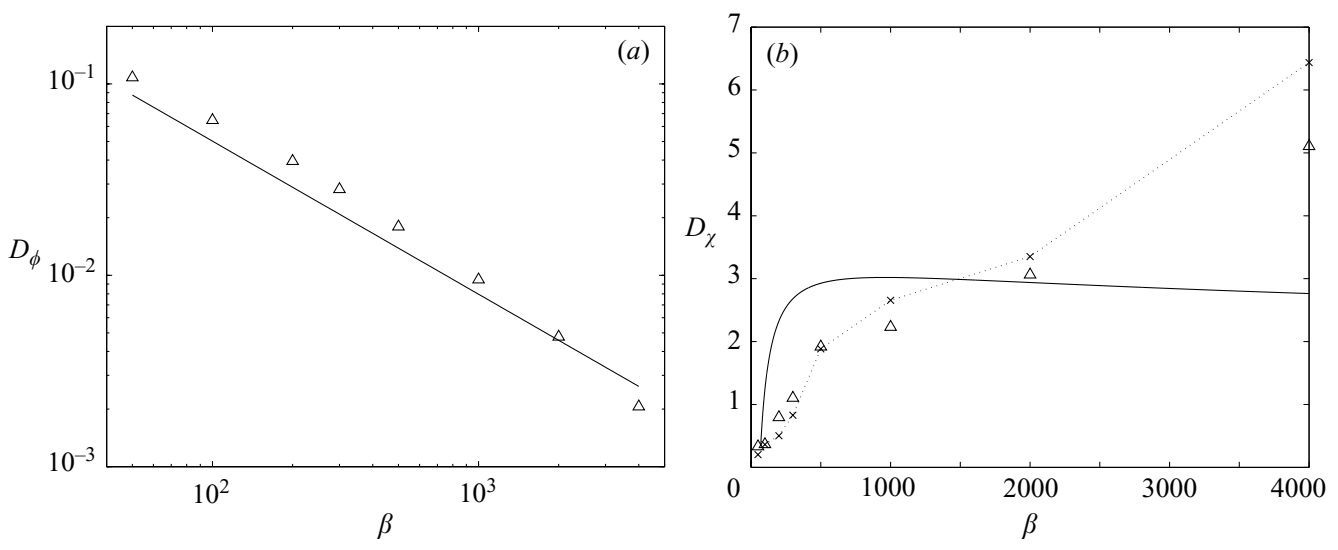

Figure 3. (a) Across-jet diffusivity $\kappa=D_{\phi}$ and $(b)$ along-jet effective diffusivity $D_{\chi}$. Triangles $(\triangle)$ represent statistics from the simulations. The solid line in $(a)$ is prediction (4.4). Solid line in (b) is theoretical fit from full scaling theory (4.9). Dotted line with $\times$-marks is semi-theoretical prediction from (3.6) using spectra of simulated $U(y)$ and simulated $D_{\phi}$, and so the values for this curve can only be calculated where simulated data exist.

transport, and so the inaccuracies in the predictions of each constituent scale will degrade the accuracy of the overall prediction. Note that all predictions shown should be inaccurate at small $\beta$, since as the anisotropy vanishes, we expect $D_{\chi} \rightarrow D_{\phi}$. This is not accounted for in the underlying shear-dispersion model (3.6).

\section{Discussion and conclusion}

As presented here, the theory applies to $\beta$-plane turbulence, but the fundamental findings are perhaps more general, potentially applicable to any flow with an isotropic turbulent background and semi-stable anisotropy in the foreground. One could thus use an independent measure of the isotropic (background) diffusivity, and the observed structure of a suitably averaged mean flow to calculate the along-jet transport via (3.6) directly (assuming a coordinate system aligned with the jet or jets), without use of the scaling theory for the jets presented in $\S 4$.

Lastly, it is worth pointing out that the theory derived above is in fact a theory for the diagonal terms of the $2 \times 2$ effective diffusivity tensor $\boldsymbol{K}$, defined by the expression $\langle\boldsymbol{u} c\rangle=\langle\boldsymbol{u} \boldsymbol{\Gamma} \cdot \boldsymbol{\chi}\rangle=-\boldsymbol{K} \boldsymbol{\Gamma}$. In this formalism, the fluxes derived above are $K^{x x}=D_{\chi}$ and $K^{y y}=D_{\phi}$. The off-diagonal terms represent components of the 'skew flux'. Rewriting the tensor as a sum of a symmetric and antisymmetric part, it can straightforwardly be shown that the antisymmetric part represents an advective transport by an incompressible eddy-induced flow. The off-diagonal terms can be calculated from the simulations described, but in order to describe their behaviour in a predictive way, care must be taken in applying the averaging operator. The total homogeneous average used above gives a single number for each simulation. The presence of stable jets, however, suggests a finer average. Preliminary results reveal an eddy-induced velocity that is of the same order, but opposed to, the mean jet flow. This will be discussed in a later paper.

I acknowledge helpful conversations with R. Ferrari and A. Majda. Although the decomposition (2.4) of the tracer is discussed in earlier references, I first learned of 
the idea from an unpublished manuscript by R. Ferrari. This work was supported by National Science Foundation grant OCE-0327470.

\section{REFERENCES}

Arbic, B. K. \& FlierL, G. R. 2004 Effects of mean flow direction on energy, isotropy, and coherence of baroclinically unstable beta-plane geostrophic turbulence. J. Phys. Oceanogr. 34, 77-93.

Barry, L., Craig, G. C. \& Thurburn, J. 2002 Poleward heat transport by the atmospheric heat engine. Nature 415, 774-777.

Bartello, P. \& Holloway, G. 1991 Passive scalar transport in $\beta$-plane turbulence. J. Fluid Mech. 223, 521-536.

Bauer, S., Swenson, M. S. \& Griffa, A. 2002 Eddy mean flow decomposition and eddy diffusivity estimates in the tropical Pacific Ocean: 2. Results. J. Geophys. Res. 107C, 3154-3172.

Bourlioux, A. \& MAJDA, A. J. 2002 Elementary models with probability distribution function intermittency for passive scalars with a mean gradient. Phys. Fluids 14, 881-897.

Chekhlov, A., Orszag, S. A., Sukoriansky, S., Galperin, B. \& Staroselsky, I. 1996 The effect of small-scale forcing on large-scale structures in two-dimensional flows. Physica D 98, 321-334.

Danilov, S. \& GryaniK, V. M. 2004 Barotropic beta-plane turbulence in a regime with strong zonal jets revisited. J. Atmos. Sci. 61, 2283-2295.

Dubus, L. 1999 Baroclinic instability of the northeast Atlantic midlatitude meridional currents: Impacts on the large-scale circulation and associated tracer mixing. $\mathrm{PhD}$ thesis, Universite de Bretagne Occidentale, Brest, France (in French).

HeLD, I. M. \& LARICHEv, V. D. 1996 A scaling theory for horizontally homogeneous, baroclinically unstable flow on a beta-plane. J. Atmos. Sci. 53, 946-952.

Holloway, G. 1986 Eddies, waves, circulation, and mixing: Statistical geofluid mechanics. Annu. Rev. Fluid Mech. 18, 91-147.

Holloway, G. \& Kristmannsson, S. S. 1984 Stirring and transport of tracer fields by geostrophic turbulence. J. Fluid Mech. 141, 27-50.

Huang, H. P., Galperin, B. \& Sukoriansky, S. 2001 Anisotropic spectra in two-dimensional turbulence on the surface of a rotationg sphere. Phys. Fluids 13, 225-240.

Klein, P., Treguier, A. M. \& HuA, B. L. 1998 Three-dimensional stirring of thermohaline fronts. J. Mar. Res. 56, 589-612.

LARICHEV, V. D. \& HeLD, I. M. 1995 Eddy amplitudes and fluxes in a homogeneous model of fully developed baroclinic instability. J. Phys. Oceanogr. 25, 2285-2297.

Majda, A. J. \& Kramer, P. R. 1999 Simplified models for turbulent diffusion: Theory, numerical modelling and physical phenomena. Phys. Rep. 314, 237.

Maltrud, M. E. \& Vallis, G. K. 1991 Energy spectra and coherent structures in forced twodimensional and beta-plane turbulence. J. Fluid Mech. 228, 321-342.

ReED, R. J. \& German, K. E. 1965 A contribution to the problem of stratospheric diffusion by large-scale mixing. Mon. Wea. Rev. 93, 313-321.

Rhines, P. B. 1975 Waves and turbulence on a $\beta$-plane. J. Fluid Mech. 69, 417-443.

Sмith, K. S. 2004 A local model for planetary atmospheres forced by small-scale convection. J. Atmos. Sci. 61, 1420-1433.

Smith, K. S., Boccaletti, G., Henning, C. C., Marinov, I. N., Tam, C. Y., Held, I. M. \& Vallis, G. K. 2002 Turbulent diffusion in the geostrophic inverse cascade. J. Fluid Mech. 469, 13-48.

Smith, R. D. \& Gent, P. R. 2004 Anisotropic Gent-McWilliams parameterization for ocean models. J. Phys. Oceanogr. 34, 2541-2564.

Spall, M. A. 2000 Generation of strong mesoscale eddies by weak ocean gyres. J. Mar. Res. 58, $97-116$.

TAYLOR, G. I. 1953 Dispersion of soluble matter in solvent flowing slowly through a tube. Proc. R. Soc. Lond. A 219, 186-203.

Young, W. R., Rhines, P. B. \& Garrett, C. J. R. 1982 Shear-flow dispersion, internal waves and horizontal mixing in the oceans. J. Phys. Oceanogr. 12, 515-527. 